\title{
SPARCL1: a potential molecule associated with tumor diagnosis, progression and prognosis of colorectal cancer
}

Hong Zhang, Emma Widegren, Da-Wei Wang and Xiao-Feng Sun

\section{Linköping University Post Print}

N.B.: When citing this work, cite the original article.

The original publication is available at www.springerlink.com:

Hong Zhang, Emma Widegren, Da-Wei Wang and Xiao-Feng Sun, SPARCL1: a potential molecule associated with tumor diagnosis, progression and prognosis of colorectal cancer, 2011, Tumour Biology, (32), 6, 1225-1231.

http://dx.doi.org/10.1007/s13277-011-0226-x

Copyright: Karger / Springer Verlag (Germany)

http://www.springerlink.com/

Postprint available at: Linköping University Electronic Press

http://urn.kb.se/resolve?urn=urn:nbn:se:liu:diva-73319 


\section{SPARCL1: a potential molecule associated with tumor}

\section{diagnosis, progression and prognosis of colorectal cancer}

Hong Zhang $^{1 *}$, Emma Widegren ${ }^{2}$, Da-Wei Wang ${ }^{3}$ and Xiao-Feng Sun ${ }^{2}$

${ }^{1}$ Division of Tumor Biology, Systems Biology Research Centre, University of

Skövde, Sweden

${ }^{2}$ Department of Oncology, Institute of Clinical and Experimental Medicine,

University of Linköping, Sweden

${ }^{3}$ Department of Stomatology, the Third Hospital of Hebei Medical University, China

Running head: SPARCL1 in Colorectal Carcinoma

Correspodence: Hong Zhang, Division of Tumor Biology, Systems Biology Research

Centre, University of Skövde, SE-54128 Skövde, Sweden. Telephone: +46-

500448482; Email: hong.zhang@ his.se 


\begin{abstract}
Background: Accumulating evidence has shown that cancers are the results of various genetic alterations. There are many genetic changes, which have been involved in tumor formation and progression in colorectal carcinomas.
\end{abstract}

Methods: In this study, we examined expression of SPARCL1 protein in the normal colorectal mucosa, adjacent normal mucosa, primary and metastatic carcinomas from colorectal cancer patients.

Results: In the same patients, we found that SPARCL1 was negative in the distant normal colorectal mucosa, weakly expressed in the adjacent normal mucosa, strongly expressed in primary colorectal adenocarcinomas, and however, slightly expressed in their metastatic tumors. The similar pattern was observed in the frequent of SPARCL1 expression from our series of colorectal cancer patients. The strongest expression and highest frequency of the SPARCL1 protein were found in the primary tumors. In the primary tumors, the frequency of SPARCL1 expression was significantly increased from the Dukes' A to Dukes' B tumors, and then decreased gradually from the Dukes' B to C and D tumors. There was no difference in the intensity of SPARCL1 expression between the central areas and invasion margins of the primary tumors. Moreover, the SPARCL1 protein was stronger expressed in the highly differentiated tumors than the lower differentiated ones. The patients with positive expression of the SPARCL1 in their tumors had worse prognosis than the patients with SPARCL-negative ones, even after the analyses by Multivariate and Interaction method. 
Conclusion: Expression of SPARCL1 protein could be used as a valuable biomarker for early diagnosis and progression in colorectal cancers, and further predicting patients' prognosis.

Keywords: SPARCL1, diagnosis, progression, prognosis, colorectal carcinoma 


\section{Background}

Colorectal cancer is one of the most common types of cancers worldwide with poor prognosis. There are more than 1 million individuals who develop colorectal cancer every year [1]. The disease-specific mortality is near one third in the developed countries although new therapeutic techniques for better treatment of the colorectal cancer patients have been introduced to the clinics during the last decades. Therefore, it appears to be important to search for molecular biomarkers for carcinogenesis, early tumor diagnosis, better treatments, and prognosis in colorectal cancers.

Colorectal cancers have been believed as phenotypes resulting from different genetic accumulations of various genetic alterations, which lead to the malignant characteristics of uncontrolled cell growth, tumor cell invasiveness, and further distant metastasis. Recent molecular biology studies on colorectal cancer have served advanced knowledge in understanding genetics of colorectal cancer [2].

Secreted Protein Acidic and Rich in Cysteine (SPARC) have been involved in many physiological functions in cell biology, such as cellular adhesion [3], cell proliferation [4], cell shape modulation, cell cycle inhibition, synthesis of extracellular matrix, and also function by interaction with extracellular matrix proteins and growth factors. In animal study, SPARC-knockout mice suffer from such as cataract due to aberrations in composition and structure of extracellular matrix [5]. Moreover, SPARC has been over-expressed in many cancer types, such as colon, and liver cancers, and has been speculated as a potential tumor suppressor, involving in cancer formation and progression [6,7]. SPARC has been associated 
with cancer chemotherapy in colon cancer, and the expression of the SPARC has been decreased after chemotherapy $[8,9]$.

There are ten protein members in the SPARC family, one of which called SPARC-like protein 1 (SPARCL1 or Hevin). SPARCL1 gene is localized at chromosome $4 \mathrm{q} 22$, and has been conserved in human. The SPARCL1 is widely expressed in normal and cancer tissues, and it is initially an anti-adhesive extracellular matrix protein with anti-proliferative effect mediated through cell-cell adhesion $[10,11]$. Down-regulation of SPARCL1 has been found in several tumor types such as pancreatic, prostate, bladder, ovarian, breast and non-small cell lung cancers and this suggests that SPARCL1 may be candidate for a tumor suppressor gene in these tumors and potential target for cancer diagnosis and therapy [12-17]. However, there is no evidence of the gene deletion or mutation that is responsible for the down-regulation of SPARCL1 in lung cancer and some other cancers $[18,19]$. Therefore, the SPARCL1 gene is unlike a classical tumor suppressor gene with a mutation in one allele and mutation or deletion in another allele. Strong SPARCL1 expression has been also observed in both tumor cells and stroma of the endometrioid adenomyfibroma. However, weak and similar staining pattern has been observed in malignant tumor and their stroma [15].

In an animal study, Lau et al. [20] have found that SPARCL1 at both RNA transcription and protein translation levels has been up-regulated in the hepatocellular cancer cells compared with the normal liver tissue, and they suggest that the SPARCL1 is related to tumor progression, and inactivation of the SPARCL1 gene is a common event in various carcinomas. Therefore, SPARCL1 has been speculated as a tumor-associated endothelium marker associated with tumor 
progression. Moreover, SPARCL1 has been proven as multifunctional glycoprotein, and involved in several process of cancer progression. However, the exact functions of SPARCL1 in human tumors progression are not completely understood, and there is no study concerning expression of SPARCL1 and its significance in colorectal cancer.

In this study, we have examined expression of SPARCL1 protein in normal colorectal mucosa, primary and metastatic colorectal cancers, and further analyzed the importance of the SPARCL1 protein expression with clinicopathological data and prognosis of the colorectal cancer patients. 


\section{Patients and Methods}

\section{Patients}

Expression of SPARCL1 proteins was examined in normal colorectal mucosa, primary, and metastatic tumors from 164 patients with primary colorectal adenocarcinoms diagnosed at the Departments of Pathology from Linköping University Hospital, Linköping, and Vrinnevi Hospital, Norrköping, Sweden, between 1982 and 2001. The normal mucosa was taken from the distant margins of surgical specimens, which showed histological free from the cancers. Adjacent normal mucosa was the tissue adjacent to the primary tumor from the same tissue sections. The metastatic tumors were from the regional lymph nodes. All patients had locally resectable colorectal adenocarcinoma, none of the patients received chemotherapy or radiotherapy before the surgery. All patients were followed-up to 10 years. Tumor localization and Dukes stages as well as local recurrence and complication were reviewed from their clinical and pathological archive. Tumor growth patterns were evaluated by two pathologists and classified as expansive and infiltrative patterns based on the patterns how cancer cell grow. Tumor differentiations were graded as better and worse. All patients were given written consent.

\section{Immunohistochemistry assay}

In this study, we attempted to clarify the role of SPARCL1 protein played in tumor diagnosis, progression, and prognosis by using corresponding distant normal mucosa, adjacent normal mucosa, primary and metastatic tumors. $3 \mu \mathrm{m}$-thick paraffin- 
embedded tissue sections were deparaffinized in xylene and rehydrated in progressively decreasing concentrations of ethanol to the water. Antigen retrieval was performed by cooking the tissue sections in $10 \mathrm{mM}$ citrate buffer in a high pressure cooker for 5 min. After cooling-down at room temperature the tissue sections were washed in deionized water and then incubated in $0.3 \% \mathrm{H}_{2} \mathrm{O}_{2} /$ methanol for $10 \mathrm{~min}$. Thereafter, the slides were placed in washing buffer $(10 \mathrm{mM}$ Tris- $\mathrm{HCl}$, $0.85 \% \mathrm{NaCl}, 0.1 \%$ bovine serum albumin, $\mathrm{pH} 7.4)$ and subjected to immunostaining. The sections were incubated with a goat polyclonal anti-SPARCL1 IgG in 1:100 dilution (R\&D Systems GmbH, Wiesbaden-Nordenstadt, Germany), or normal goat IgG (DAKO Corporation, Denmark) as negative controls. The slides were then rinsed with washing buffer and incubated with anti-goat IgG HRPO-conjugated (Santa Cruz Biotechnology Inc., Santa Cruz, CA, USA) for $1 \mathrm{~h}$ at room temperature. The tissue sections were then washed in washing buffer and incubated with DABchromogen substrate mixture (DAKO). Finally, sections were slightly counterstained with hematoxylene, dehydrated and mounted under cover slips. In order to minimize subjective bias, the staining intensity and staining patterns were separately scored by two authors (HZ and DW). The samples were scored as SPARCL1 positive expression when the number of positive cells was more than $10 \%$ of the total cells counted regardless of the staining intensity. The sections with discrepant were re-examined until consensus reached.

\section{Statistical analysis}

The statistical analyses were performed with STATISTICA program (Version 9.0). The differences in frequency of SPARCL1 expression between the normal mucosa, primary and metastatic tumors were tested by the Chi square or McNemar's method. 
The relationship between the SPARCL1 expression and clinicopathological was analyzed by the Chi-square. The relationship between SPARCL1 expression and survival was tested by Cox's proportional hazard model. The Kaplan-Meier method was used to calculate survival curves. The patients' survivals were further analyzed by Multivariate and Interaction methods. All $p$ values were two sided, and $p<0.05$ was considered as statistically significant. 


\section{Results}

\section{Expression of SPARCL1 protein in normal colorectal mucosa and colorectal adenocarcinomas}

We examined expression of SPARCL1 protein by immunohistochemistry and found that in the matched samples from the same patients, SPARCL1 protein was negative in the distant normal colorectal mucosa (Figure 1A) and even in adjacent mucosa Figure 1B). However, the SPARCL1 was strongly expressed in their primary colorectal carcinomas (Figure 1C) and weakly expressed in their metastatic tumors in the lymph nodes (Figure 1D). In the primary tumors, expression of the SPARCL1 protein was stronger in the high-differentiated tumors (Figure 2A) than those in the low differentiated ones (Figure 2B). We also compared the intensity and patterns of the SPARCL1 protein expression in the tumor central areas (Figure 2C) and invasion margins (Figure 2D) of the primary adenocarcinomas, and did not find the difference between the different areas. Moreover, there were similar expression patterns of the SPARCL1 protein of the stroma in both normal (Figure 2E) and tumor (Figure 2F) samples. When frequency of the SPARCL1 protein expression was further analyzed in the whole series samples in our patients the similar expression pattern was found as in the staining expression in the matched samples. The frequency of SPARCL1 expression was $17 \%$ in the distant normal colorectal mucosa, $19 \%$ in the adjacent normal mucosa, $80 \%$ in the primary colorectal cancer, and $41 \%$ in the lymph metastasis (Figure 3). 

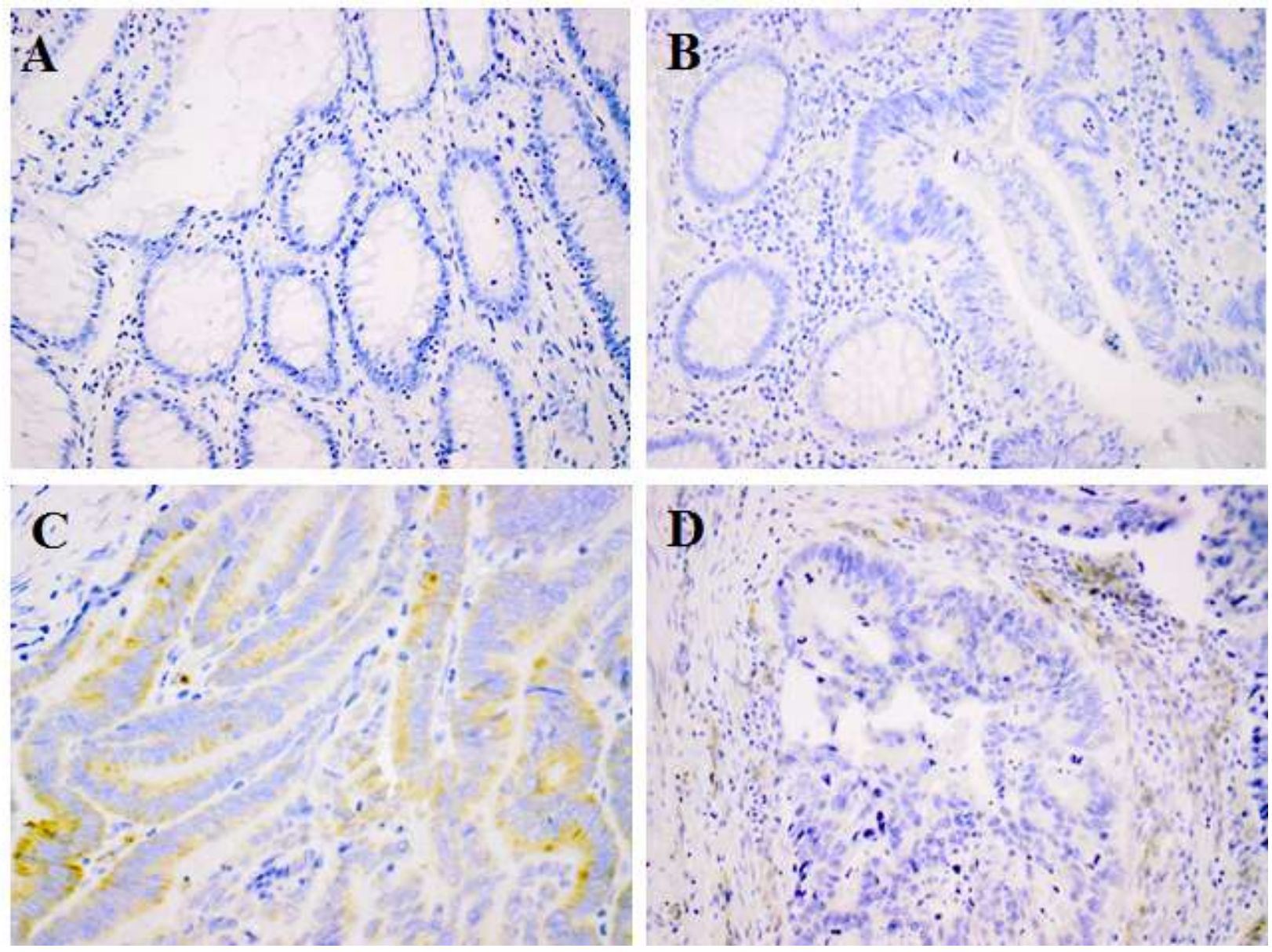

Figure 1. Expression of SPARCL1 protein in the normal colon mucosa, adjacent normal mucosa, primary colorectal carcinoma and lymph node metastasis from the same cancer patient. The SPARCL1 protein was negative in the normal colorectal mucosa (A), and in the adjacent colorectal mucosa (B). The SPARCL1 protein was strongly expressed in the cytoplasm of colorectal cancer cells (C), and weakly expressed in the metastatic cancer cells (D).

\section{Expression of SPARCL1 protein and clinicopathological features of colorectal cancer patients}

Associations of expression of the SPARCL1 protein in the primary colorectal carcinomas with clinicopathological features were further analyzed. The expression of SPARCL1 protein was found to be associated with Dukes' stages $(p=0.005)$. The frequency of SPARCL1 expression was significantly increased from the Dukes' A to Dukes' B tumors, and then decreased gradually from the Dukes' B to C and D 

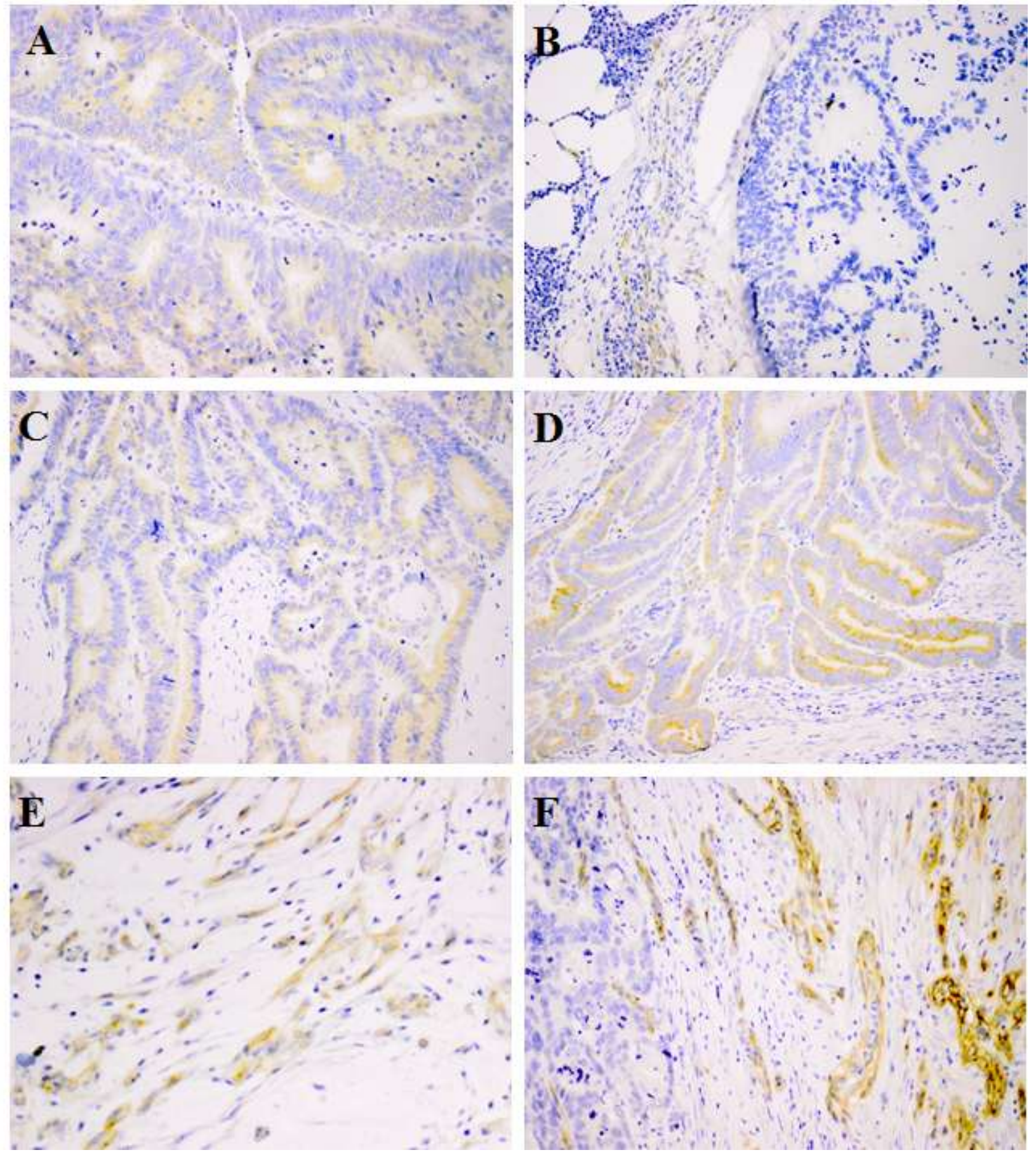

Figure 2. Expression of SPARCL1 protein in the primary colorectal carcinomas. The SPARCL1 was positive in the high-differentiated colorectal adenocarcinoma (A), and negative in the low differentiated colorectal adenocarcinoma (B). A similar expressed pattern of SPARCL1 protein was found in both central part (C) and invasive margins (D) of the primary tumors. The SPARCL1 protein was strongly expressed in the endothelial cells of the stromal blood vessels in both normal (E) and cancer $(\mathrm{F})$ stroma. 


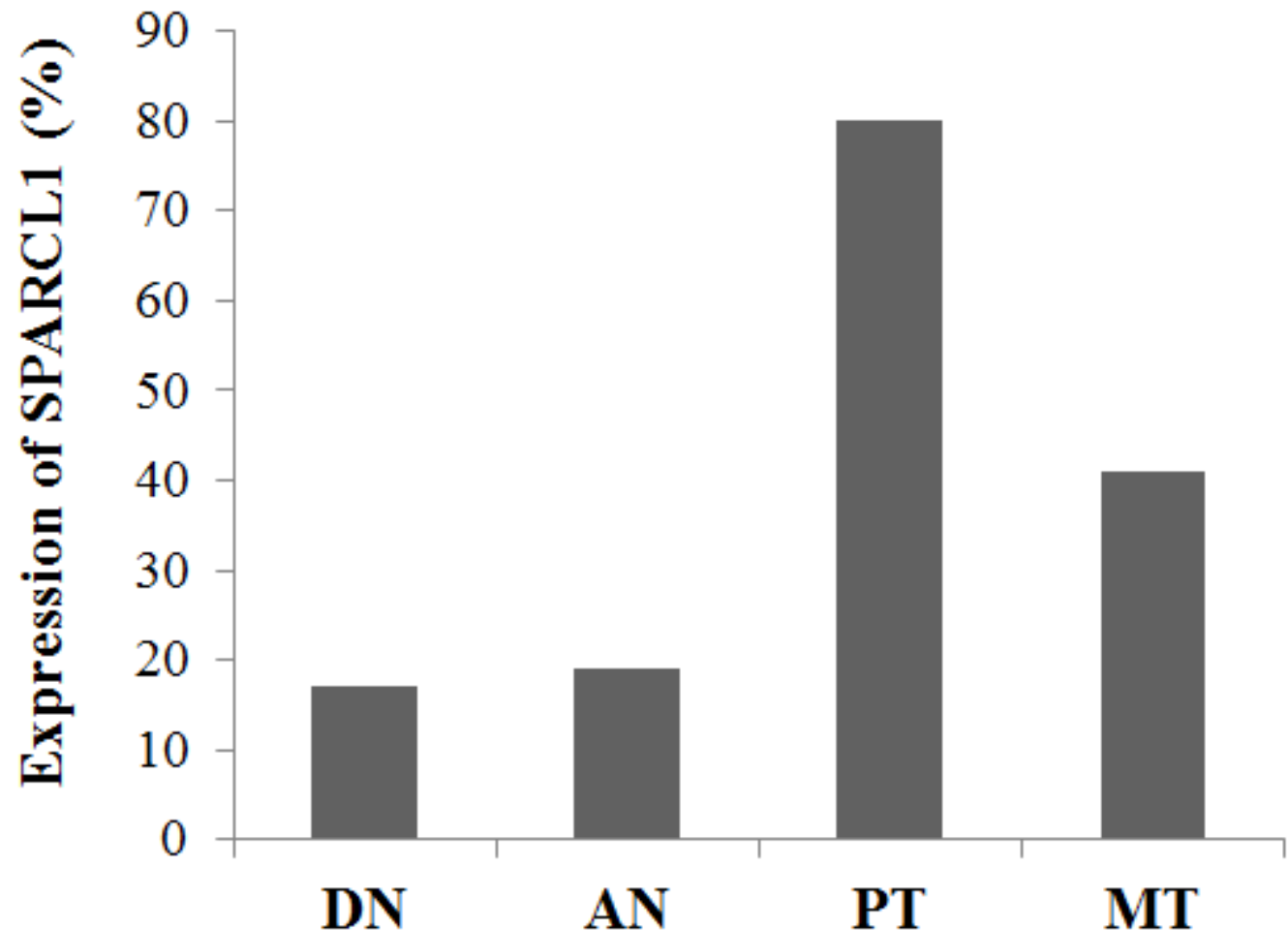

Figure 3. Frequency of SPARCL1 expression in colorectal distant normal (DN) and adjacent normal (AN) mucosa, as well as primary colorectal tumors (PC) and lymph node metastatic tumors (MT). The expression frequency was significantly increased from the normal colorectal mucosa to the primary colorectal cancer, and the frequency was significantly decreased from the primary cancer to metastases.

tumors (Figure 4). The SPARCL1 protein was stronger expressed in the highly differentiated tumors than the lower differentiated ones $(\mathrm{p}=0.01)$. However, there were no difference with patients' gender, age, tumors location, growth patterns, tumor local recurrence and complication of the patients $(p>0.05$, Table 1$)$.

\section{Expression of SPARCL1 protein and patients survival}

As shown in Figure 5, we further analyzed the association of SPARCL1 protein expression with patients' outcome, and found that the patients with positive expression of the SPARCL1 protein in their primary tumors had short survival after 


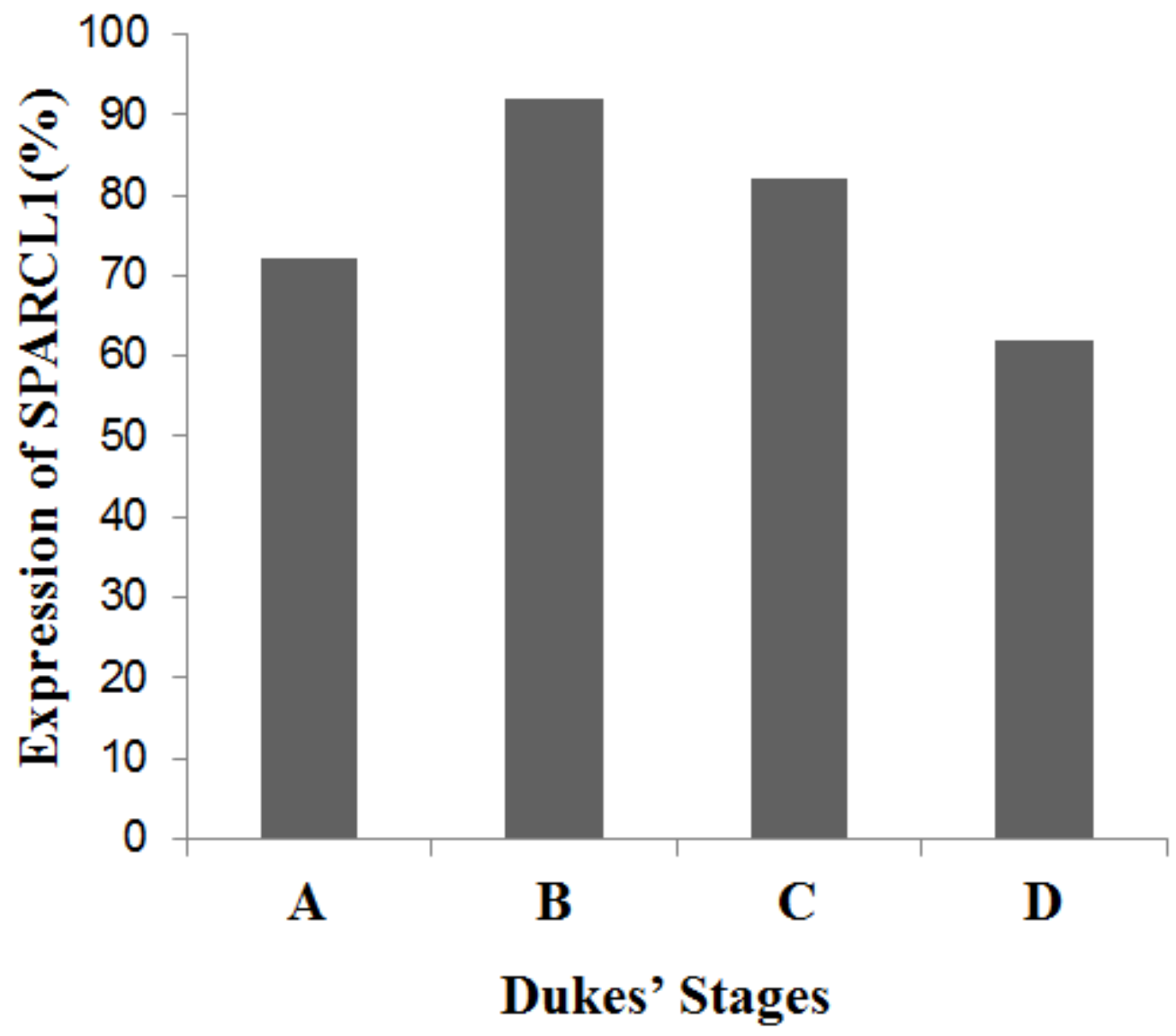

Figure 4. Expression of SPARCL1was associated with Dukes' stages. Frequency of SPARCL1 expression was significantly increased from Dukes' stage A to B, and decreased from Dukes' B tumors to C and D tumors.

the surgical treatment than those patients with negative tumors $(\mathrm{p}=0.017)$. The difference still existed as statistical significance even the value was adjudged with Dukes' stages $(\mathrm{p}=0.01)$, tumor differentiations $(\mathrm{p}=0.047)$ and both together Dukes' stages and tumor differentiations $(\mathrm{p}=0.006)$ by Multivariate and Interaction analyses . 
Table 1. The relationship of SPARCL1 expression of primary tumors with clinicopathological variables

\begin{tabular}{|c|c|c|c|}
\hline \multirow[b]{2}{*}{ Variables } & \multicolumn{2}{|c|}{ SPARCL1 expression } & \multirow[t]{2}{*}{ Pvalue } \\
\hline & Negative $(\%)$ & Positive (\%) & \\
\hline Gender & & & 0.56 \\
\hline Male & $19(22)$ & $68(78)$ & \\
\hline Female & $14(18)$ & $63(82)$ & \\
\hline Age & & & 0.49 \\
\hline$<71$ & $15(23)$ & $51(77)$ & \\
\hline$\geq 71$ & $18(18)$ & $80(82)$ & \\
\hline Tumor location & & & 0.37 \\
\hline Right colon & $12(27)$ & $33(73)$ & \\
\hline Left colon & $5(15)$ & $28(85)$ & \\
\hline Rectum & $15(18)$ & $69(82)$ & \\
\hline Dukes stage & & & 0.005 \\
\hline A & $7(28)$ & $18(72)$ & \\
\hline B & $5(8)$ & $57(92)$ & \\
\hline $\mathrm{C}$ & $8(18)$ & $36(82)$ & \\
\hline $\mathrm{D}$ & $12(38)$ & $20(62)$ & \\
\hline Growth pattern & & & 0.13 \\
\hline Expansive & $15(16)$ & $79(84)$ & \\
\hline Infiltration & $17(26)$ & $49(74)$ & \\
\hline Differentiation & & & 0.01 \\
\hline Better & $17(15)$ & $97(85)$ & \\
\hline Worse & $16(32)$ & $34(68)$ & \\
\hline Complication & & & 0.07 \\
\hline No & $11(23)$ & $36(77)$ & \\
\hline Yes & $4(9)$ & $39(91)$ & \\
\hline Local recurrence & & & 0.38 \\
\hline No & $13(16)$ & $70(84)$ & \\
\hline Yes & $2(29)$ & $5(71)$ & \\
\hline
\end{tabular}




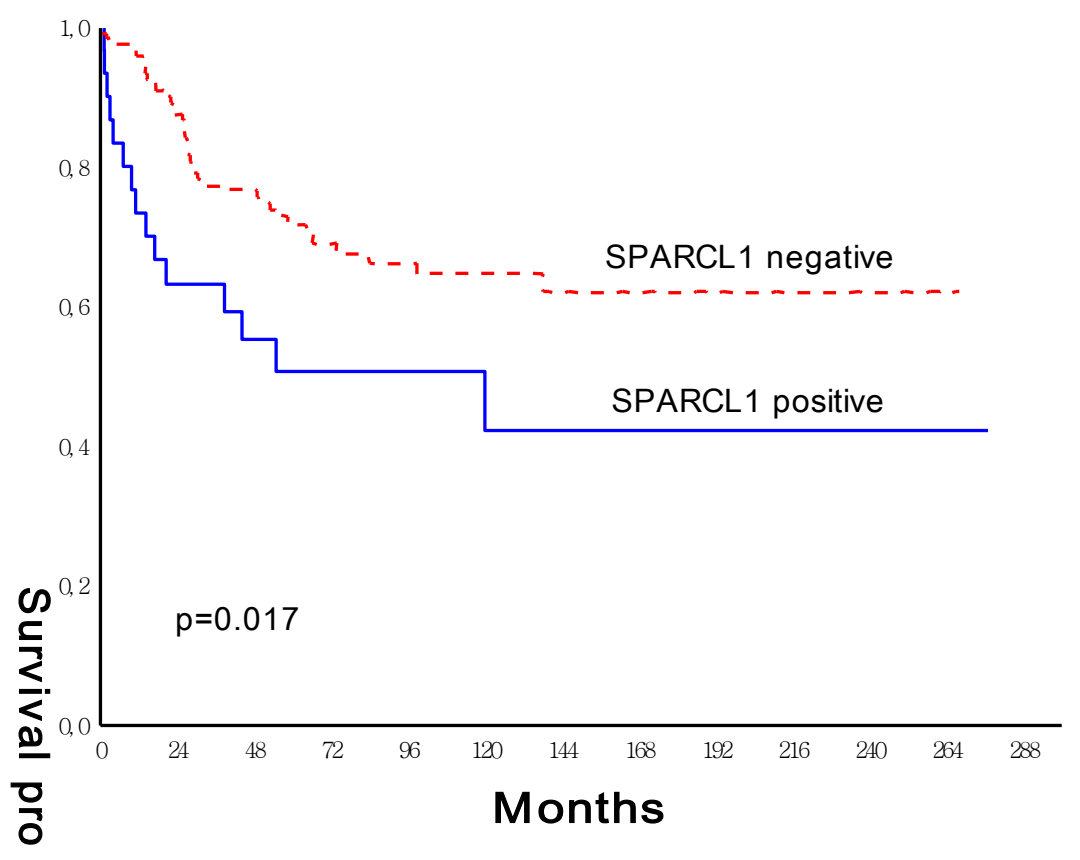

Figure 5. Expression of SPARCL1 in the primary colorectal cancer was positively correlated to the patients' survival. The patients with positive expression of the SPARCL1 protein had significantly worse prognosis as compared to the patients with SPARCL1 negative tumors.

\section{Discussion}

In this study, we examined expression of SPARCL1 protein in the normal colorectal mucosa, adjacent mucosa, primary and metastatic colorectal adenocarcinomas in colorectal cancer patients, and further analyzed the associations of the SPARCL1 expression with clinocopathlogical features in colorectal cancer patients. We found that in the same patient SPARCL1 protein was negative in both normal colorectal mucosa and adjacent mucosa. However, the SPARCL1 protein was strongly expressed in their matched primary tumor and weakly expressed in the metastatic tumors in the local lymph nodes. These results clearly demonstrated that SPARCL1 protein was up-regulated from the normal colorectal mucosa to the primary colorectal adenocarcinoma. Since it was markedly decreased from the primary tumors to the metastases it was believed as an early event and played an essential 
role during the colorectal cancer early development and progression. SPARCL1 protein might be used as a valuable biomarker for early diagnosis of colorectal cancer, and we further proposed that SPARCL1 protein might function as an "oncoprotein" during progression of colorectal cancer which was in the same line as it is speculated in hepatocytal cancers showing that both SPARC and Hevin have been elevated at both mRNA and protein levels. They play an important role in hepatocytal cancer progression [20]. However, in a study of non-small cell lung carcinoma, the SPARCL1 at mRNA levels was found to be down-regulated. Moreover, it is unlike a classical tumor suppressor gene with a deletion or mutation in one allele and mutation in another allele. The authors suggest that the downregulation of SPARCL1 mRNA in lung cancer may be mediated through other transacting factors [19]. SPARCL1 has been shown to inhibit cellular progression from G1 to S phase or prolong G1 phase, and it is strongly down-regulated in most malignant cells [12]. Therefore, SPARCL1 protein may play opposite roles through different mechanisms in different tumor types as either "oncogene" colorectal carcinoma or "tumor suppressor" in lung cancer.

In this study, we found that in the primary colorectal cancers the expression of SPARCL1 protein was related to Dukes' stages. The SPARCL1 expression was increased from Dukes' stages A to B, and then decreased from Dukes' stages B to C and D stages, indicating that the expression of SPARCL1 protein was an early event during the cancer development, and could be used as a valuable biomarker for colorectal cancer staging. Dukes staging in colorectal cancer is one of the most powerful prognostic factors to predict the patients' outcome. The patients in earlier stages have better survival. The prognosis for the colorectal cancer patients in Dukes' stage A has been significantly better that the patients with Dukes' stage B tumors. 
However, it is practically extremely difficult to make a clear-cut between Dukes' stage A and B, and even lymph node micro-metastases are carefully examined to differentiate Dukes' stage A from Dukes' stage B. The lymph node micro-metastases have not found to be a useful prognostic marker and do not imply different strategies for additional therapy or follow-up in colorectal cancer patients [21]. The result from this study could give an additional evidence for the clear-cut between Dukes' stage A and B.

We found that expression of SPARCL1 protein was related to patients' survival. Over-expression of SPARCL1 protein in the primary colorectal carcinomas indicated that the patients had a short survival. On the other hand, the patients had better outcome when they had SPARCL1-negative tumors. The statistical significance remained even after the analyses with Multivariate and Interaction method. The result further provided an evidence for the SPARCL1 protein as an "oncoprotein" in colorectal cancers, and it promoted the early formation of colorectal carcinomas. We proposed that SPARCL1 protein might be utilized as a valuable indicator for predicting patients' outcome in colorectal carcinomas.

\section{Conclusion}

In summary, SPARCL1 protein was over-expressed in the early stages of primary colorectal carcinomas. The expression of SPARCL1 in the primary colorectal cancer was associated with worse prognosis for colorectal cancer patients (Figure 6). 


\section{Conflicts of interest}

None declared.

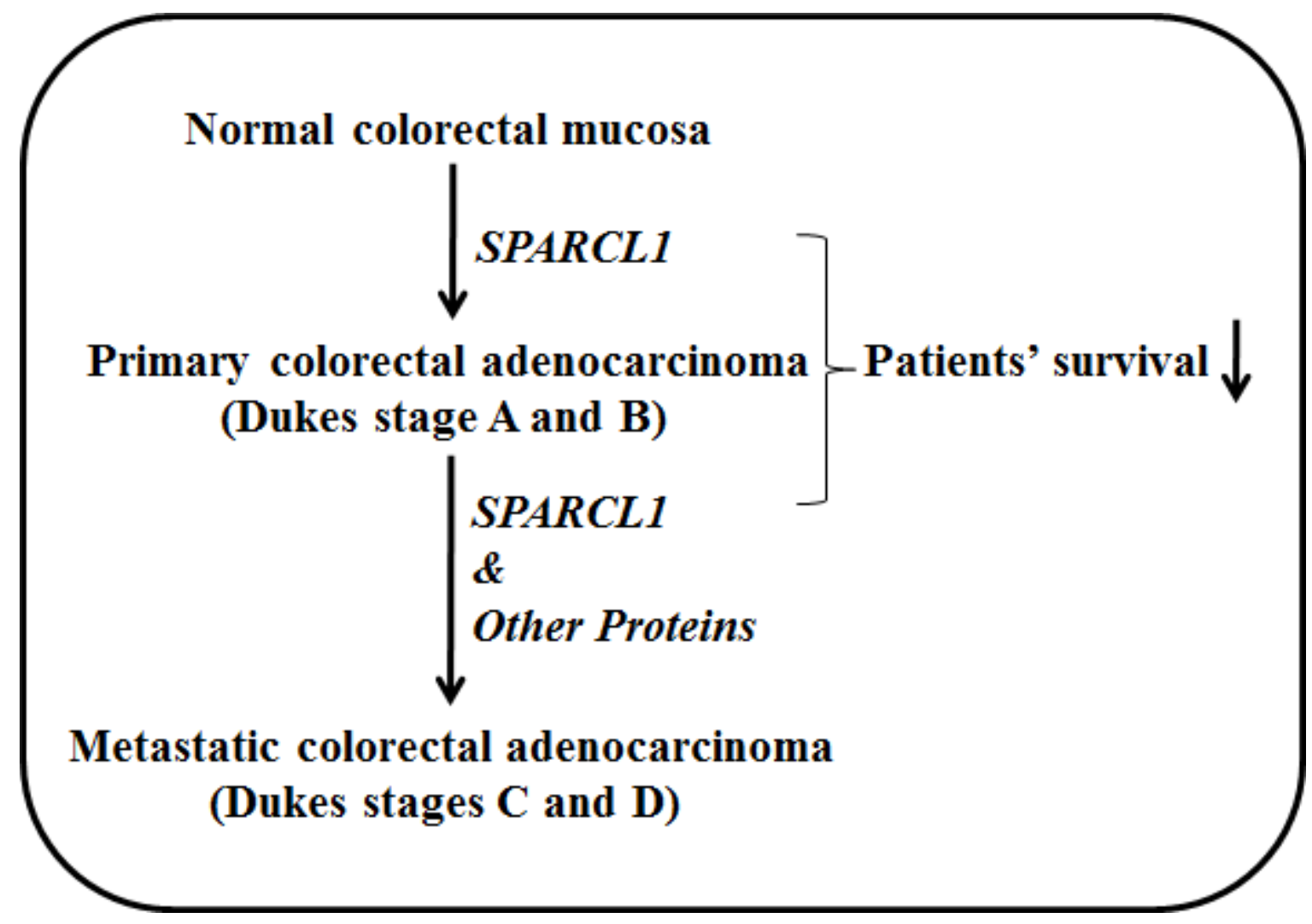

Figure 6. SPARCL1 protein as an "oncoprotein" played an essential role in the early development of colorectal cancer, and patients' survival. 


\section{Acknowledgements}

The authors thank Helen Richard, Cecilia Bergenwald, Gertrud Stridh, Gunnel Lindell and Kerstin Ingels, Department of Pathology, Linköping University Hospital, Sweden, for kindly preparing tissue sections. The study was supported by the grants from the Swedish Cancer Foundation, Swedish Research Council and the Health Research Council in the South-East of Sweden. 


\section{References}

1. Jemal A, Siegel R, Ward E, Hao Y, Xu J and Thun MJ: Cancer statistics, 2009. CA Cancer J Clin 59: 225-249, 2009.

2. Midgley R, Rasul K, Al Salama H and Kerr DJ: Gene profiling in early stage disease. Cancer J 16: 210-213, 2010.

3. Murphy-Ullrich JE: The de-adhesive activity of matricellular proteins: is intermediate cell adhesion an adaptive state? J Clin Invest 107: 785-790, 2001.

4. Kouros M: SPARC (osteonectin/BM-40). Int J Biochem Cell Biol 31: 13631366, 1999.

5. Brekken RA, Sullivan MM, Workman G, et al: Expression and characterization of murine hevin (SC1), a member of the SPARC family of matricellular proteins. J Histo Cytochem 52: 735-748, 2004.

6. Sullivan MM and Sage EH: Hevin/SC1, a matricellular glycoprotein and potential tumor-suppressor of the SPARC/BM-40/Osteonectin family. Int J Biochem Cell Biol 36: 991-996, 2004.

7. Framson PE and Sage EH: SPARC and tumor growth: where the seed meets the soil? J Cell Biochem 92: 679-690, 2004.

8. Tai IT and Tang MJ: SPARC in cancer biology: its role in cancer progression and potential for therapy. Drug Resistance Updates 11: 231-246, 2008.

9. Mintz MB, Sowers R. Brown KM, et al: An Expression Signature Classifies Chemotherapy-Resistant Pediatric Osteosarcoma. Cancer Res 65: 1748-1754, 2005. 
10. Hambrock HO, Nitsche P, Hansen U, et al: SC1/Hevin: an extracellular calcium-modulated protein that binds collagen. J Biol Chem 278: 11351-11358, 2003.

11. Girard JP and Springer TA: Modulation of endothelial cell adhesion by hevin, an acidic protein associated with high endothelial venules. J Biol Chem 271: 4511-4517, 1996.

12. Claeskens A, Ongenae N, Neefs JM, et al: Hevin is down-regulated in many cancers and is a negative regulator of cell growth and proliferation. $\mathrm{Br} \mathrm{J}$ Cancer 82: 1123-1130, 2000.

13. Nelson PS, Plymate SR, Wang K, et al: Hevin, an antiadhesive extracellular matrix protein, is down-regulated in metastatic prostate adenocarcinoma. Cancer Res 58: 232-236, 1998.

14. Esposito I, Kayed H, Keleg S, et al: Tumor-suppressor function of SPARC-like protein 1/hevin in pancreatic cancer. Neoplasia 9: 8-17, 2007.

15. Biade S, Marinucci M, Schick J, et al: Gene expression profiling of human ovarian tumours. Br J Cancer 95: 1092-1100, 2006.

16. Bergamaschi A, Tagliabue E, Sørlie T, et al: Extracellular matrix signature identifies breast cancer subgroups with different clinical outcome. J Pathol 214: 357-367, 2008.

17. Isler SG, Schenk S, Bendik I, et al: Genomic organization and chromosomal mapping of SPARC-like 1, a gene down regulated in cancers. Int J Oncol 18: 521-526, 2001. 
18. Bendik I, Schraml P and Ludwig CU: Characterization of MAST9/Hevin, a SPARC-like protein, that is down-regulated in non-small cell lung cancer. Cancer Res 58: 626-629, 1998.

19. Isler SG, Ludwig CU, Chiquet-Ehrismann R and Schenk S: Evidence for transcriptional repression of SPARC-like 1, a gene downregulated in human lung tumors. Int J Oncol 25: 1073-1079, 2004.

20. Lau CPY, Poon RTP, Cheung ST, Yu WC and Fan ST: SPARC and hevin expression correlate with tumour angiogenesis in hepatocellular carcinoma. $\mathbf{J}$ Pathol 210: 459-468, 2006.

21. Oberg A, Stenling R, Tavelin B and Lindmark G: Are lymph node micrometastases of any clinical significance in Dukes Stages A and B colorectal cancer? Dis Colon Rectum 41: 1244-1249, 1998. 